\title{
Glioblastoma multiforme with long term survival
}

\author{
Prabal Deb, Mehar Chand Sharma, Ashok Kumar Mahapatra*, Deepak Agarwal ${ }^{\star}$, Chitra Sarkar \\ Departments of Pathology and Neurosurgery*, All India Institute of Medical Sciences, New Delhi, India
}

\begin{abstract}
Glioblastoma multiforme (GBM) Patients generally have a dismal prognosis, with median survival of 10-12 months. GBM with long-term survival (LTS) of $\geq 5$ years is rare, and no definite markers indicating better prognosis have been identified till date. The present study was undertaken to evaluate GBMs with LTS in order to identify additional correlates associated with favourable outcome.

The cases were evaluated for relevant clinicopathological data, proliferation index and expression of tumortumour suppressor gene ( $p 53)$, cyclin-dependant kinase-inhibitors ( $p 27$ and $p 16)$ and epidermal growth factor receptor (EGFR) proteins.
\end{abstract}

Six cases of GBM with LTS with an average survival of 9 years (range 5-15 years) were identified. All were young patients with mean age of 27 years (range 8-45 years). Histology of three cases was consistent with conventional GBM, while two showed prominent oligodendroglial component admixed with GBM areas. One was a giant cell GBM, which progressed to gliosarcoma on recurrence. The mean MIB$1 \mathrm{LI}$ was $12 \%$ (range $6-20 \%$ ). p53 was immunopositive in 4 out of 5 cases. EGFR and $p 27$ were immunonegative in all, whereas $p 16$ was immunonegative in 3 out of 5 cases.

Currently, in the absence of specific molecular and genetic markers, GBM in young patients should be meticulously evaluated for foci of oligodendroglial component and/or giant cell elements, in addition to proliferative index and p53 expression, since these probably have prognostic connotations, as evident in this study. The role of $p 16$ and $p 27$ however needs better definition with study of more number of cases.

Key words : Glioblastoma multiforme, long-term survival, favourable outcome

\section{Introduction}

Glioblastoma multiforme (GBM) are generally associated with a poor prognosis, with median survival of 10-12 months. ${ }^{[1]} 5$ year survival is rare and is reported to be around $4-5 \%$ only. ${ }^{[2,3]}$

Owing to the paucity of GBMs with long-term survival (LTS), no definite picture has emerged till date, though a number of clinical, ${ }^{[4]}$ histopathological ${ }^{[5,6]}$ and molecular markers $^{[5,7,8]}$ have been associated with favourable prognosis.

It was aimed to evaluate GBMs with LTS for their distinctive clinical features, histopathological profile, proliferation index and expression of tumour suppressor gene (p53), cyclindependant kinase (cdk)-inhibitors ( $p 2 \%$ and $p 16)$ and epidermal growth factor receptor (EGFR) proteins, in order to identify any additional correlates associated with LTS.

\section{Case series}

Out of a total of 1296 cases of GBM diagnosed during 19891999,6 cases $(0.5 \%)$ with survival $\geq 5$ years, were selected from the records.

\section{Clinical profile (Table1)}

All were young patients with mean age of 27 years (range 845 years) and an equal male : female ratio. Frontal lobe was the commonest site affected. All the cases underwent gross total resection, with postoperative chemotherapy and radiotherapy.

\section{Histopathological evaluation}

H\&E stained slides were reviewed and diagnosis reconfirmed using the WHO (2000) $)^{[1]}$ criteria. Representative blocks of formalin-fixed paraffin-embedded tissue, where available, were selected, 55-mm sections cut and immunohistochemical staining performed by LSAB technique (LSAB Kit, Dakopatts,) using monoclonal antibodies to p53 (1:100, SantaCruz), p16 (1:400, Neomarkers), p2\% (1:25, Dakopatts), EGFR (1:50, Neomarkers) and glial fibrillary acidic protein (GFAP 1:1000, Dakopatts). 


\begin{tabular}{ll}
\hline \multicolumn{1}{c}{ Table 1: Summary of clinical profile of GBM cases with } \\
long-term survival
\end{tabular}

Current status: all alive/on regular follow up.

In case 6 , special stains for reticulin (Gomori-Sweet silver stain) and Masson's trichrome, as well as IHC using monoclonal antibodies to vimentin (1:50, Novacastra), CD 34 (1:300, Dakopatts), Desmin (1:15, Dakopatts) and smooth muscle actin (SMA - 1:15, Dakopatts) were done.

Proliferation index was evaluated using MIB-1 antibody (1:200, Dakopatts), and manually counting 1000 cells from at least 5 representative microscopic fields, at high power magnification $(400 \mathrm{x})$.

\section{Histopathological features (Table 2)}

Three cases (cases 1,3,5) were conventional GBMs, while 2 (cases 2 and 4) showed focal areas of oligodendroglial differentiation admixed with GBM [Figure 1A,B], with extensive areas of GFAP immunopositivity.

Case 6 was a giant cell GBM (GCG), with two recurrences

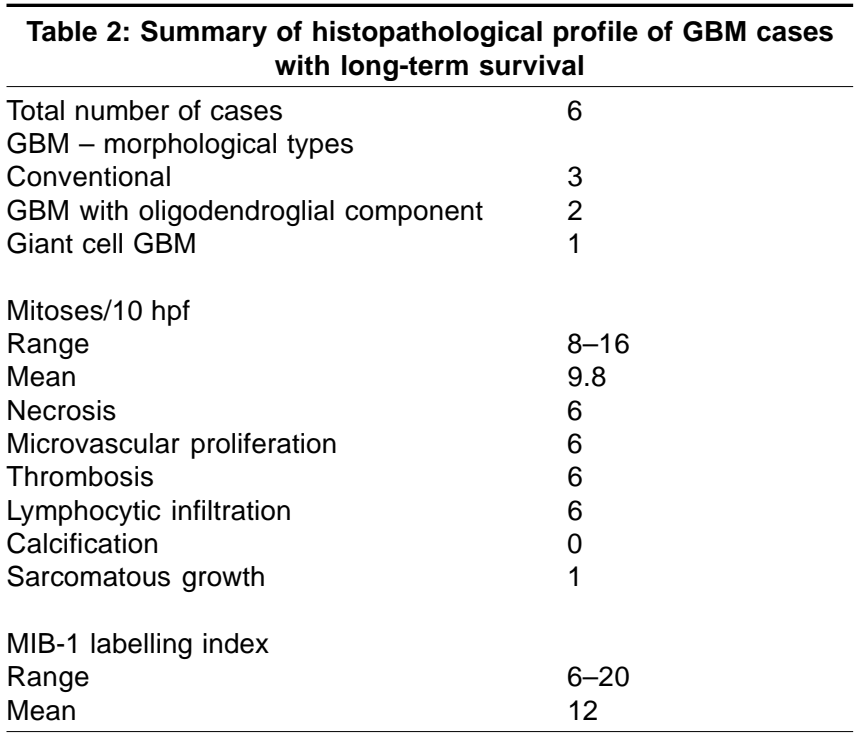

at 8 and 9 years after initial surgery. Morphology at initial surgery and first recurrence was that of GCG [Figure 2A,B], characterized by monstrous multinucleated giant cells with abundant eosinophilic cytoplasm and a reticulin-poor morphology. Biopsy at second recurrence showed a gliosarcoma with distinct GFAP-immunopositive giant cell GBM areas, and reticulin-rich GFAP-immunonegative sarcomatous areas composed of densely packed long bundles of spindle cells, with nuclear atypia and high mitotic activity. Immunostaining for CD34, SMA and desmin were however negative in both the initial and recurrent tumours.

Mitoses ranged from 8-16/10 high power field (hpf) (mean $9.8 / 10 \mathrm{hpf})$.

\section{MIB-1 LI}

Proliferation index in the five cases, as reflected by MIB1LI, ranged between $6-20 \%$ with a mean of $12 \%$ [Table 2 ].

\section{Expression of oncogene / tumour suppressor gene protein}

p53 was immunopositive in $4 / 5$ cases [Figure $3 \mathrm{~A}$ ], the exception being case 5 (conventional GBM), while $p 16$ was immunonegative in $3 / 5$ cases (barring two cases of conven-

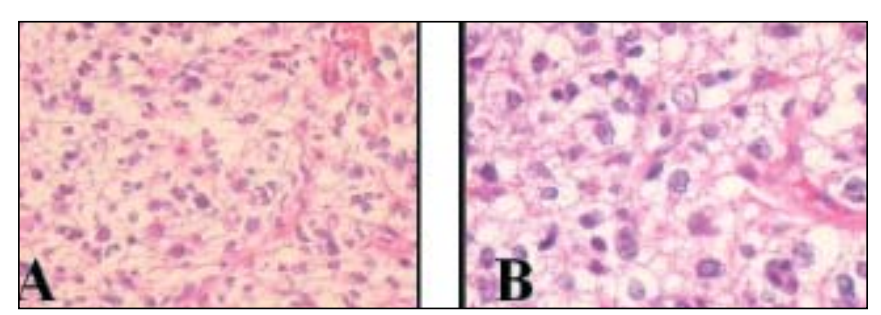

Figure 1: Photomicrographs of case 4 - -showing GBM with admixed oligodendroglial component (A: H\&E x200) and (B: H\&E x400).

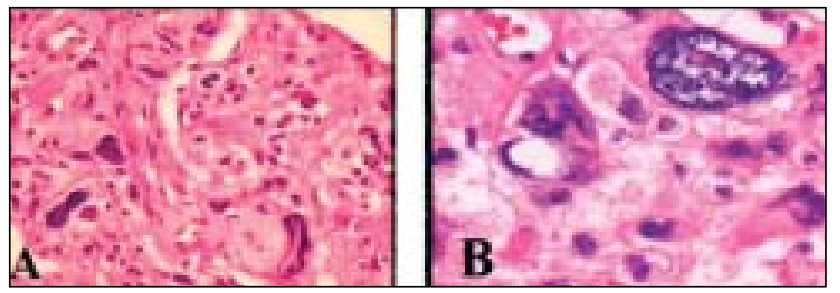

Figure 2: Photomicrographs of the initial tumour of case 6 - showing giant cell GBM with numerous bizarre multinucleated giant cells ( $A$ : $H \& E \times 200)$ and (B: H\&E x400).

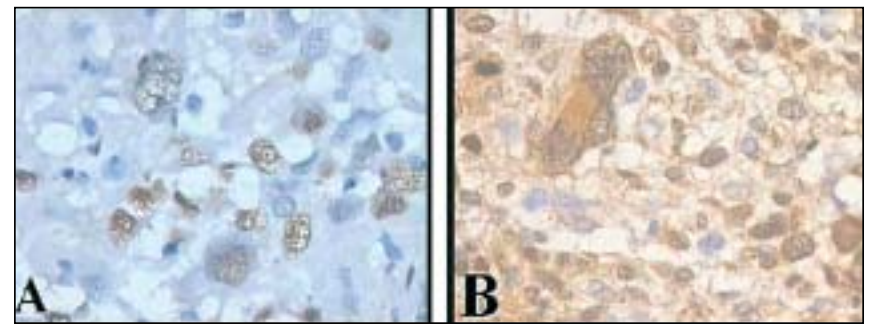

Figure 3: Photomicrograph of the GBM with long survival (case3), showing nuclear immunopositivity for p53 (A: p53 x400) and nuclear and cytoplasmic immunopositivity for p16 (B: $p 16 \times 400)$ 
tional GBM) [Figure 3B].

However, all the cases were immunonegative for EGFR and p2\%.

\section{Follow up}

On regular follow up, it was observed that all the patients are currently alive, with mean survival of 9 years (range 5-15 years). The case of GCG and two cases of conventional GBM (cases 1,5) had recurrences.

\section{Discussion}

Till date, little data is available regarding GBM patients with LTS ( $\geq 5$ years). Scott et al ${ }^{[7]}$ analysed 689 GBMs and confirmed only $2.2 \%$ to have LTS, while, McLendon et al ${ }^{[2]}$ detected 2\% GBM with LTS among 766 cases. Recently, Shinojima et al ${ }^{[4]}$ reported 6 such cases $(5 \%)$ out of 113 adult patients with GBM. However, this study analysed the first such data in India and observed an incidence of only $0.5 \%$.

On analysing the available literature, it was observed that clinical parameters like young age,${ }^{1}$ predominance of female sex ${ }^{[4]}$ aggressive surgical resection and a good Karnofsky performance score ${ }^{[5]}$ in GBM are generally associated with better prognosis. Similarly, in the present study cases were young (mean age 27 years), lacked a male predominance and had undergone gross total resection. This was however different from that observed in conventional GBMs.

Typically, the histological predictors of better outcome in GBMs include presence of giant cells, ${ }^{[2,4,6]}$ focal oligodendroglial differentiation, ${ }^{[2]}$ absence of small anaplastic cells ${ }^{[2]}$ and evidence of better differentiation with more GFAP positivity, ${ }^{[5]}$ all of which were evident in the present series.

GBMs with LTS characteristically demonstrate a lower proliferation index (MIB-1LI 5.7\% ${ }^{[8]}$ as compared to the WHO recommended levels of $15-20 \%$ for conventional GBM. ${ }^{[1]}$ Overall, the proliferative activity in the present series was $12 \%$ (range 6-20\%), which was not only lower than the $\mathrm{WHO}^{[1]}$ recommended level, but was also substantially lower than the mean cut-off level $(22.09 \pm 14.73)^{[9]}$ for conventional GBMs in this laboratory.

Although a number of molecular markers have been studied for prognostication in GBMs, only a combination of LOH $1 p$ and $19 q$ defined GBM patients with a significantly better survival. ${ }^{[5]}$ However, Burton et al ${ }^{[8]}$ evaluated the largest sample size of GBM with LTS and observed differences in the rate of abnormal $p 53$ expression (85\%), when compared with tumours from a cohort of patients with similar age and KPS but with poor survival. In the present study, $p 53$ was immunopositive in 4/5 cases, while that for conventional GBMs at this centre ranged from $43-61 \%$ (mean 50\%). ${ }^{[10]}$ Though these observations suggest that the LTS group may possibly represent a different subgroup of GBMs with favourable outcome, further evaluation with more number of cases is warranted for a definite opinion.

Burton et al ${ }^{[8]}$ evaluated EGFR as a prognostic marker in gliomas, and observed over expression in 12\% of GBMs with LTS as compared to $25 \%$ of conventional GBMs. This difference was not statistically significant, and hence no consensus has been reached in regard to its significance ${ }^{[8]} \mathrm{A}$ recent study by Heimberger et al ${ }^{[11]}$ has reported EGFR over expression in 50-60\% of GBM, while the most common mutant EGFRvIII expression in 24-67\% cases, but failed to find a correlation with overall survival. However, in cases surviving $>1$ year, EGFRvIII was an independent negative prognostic indicator. In the present study all cases were immunonegative for EGFR and hence were non-contributory.

Schmidt et $\mathrm{al}^{[5]}$ observed LOH $1 \mathrm{p} / 19 \mathrm{q}$ in 5 cases of primary GBM, which did not exhibit any areas of oligodendroglial differentiation, but had a significantly better survival. Since all the cases in the present study were possibly de novo GBMs (with no clinical or histopathological evidence of progression from low-grade astrocytomas) with a better survival, molecular evaluation with $\mathrm{LOH} 1 \mathrm{p} / 19 \mathrm{q}$ studies, becomes imperative to exclude foci of oligodendroglial alterations in the remaining cases (omitted owing to lack of facility in this centre).

Studies with cyclin-dependant kinase-inhibitors ( $p 16$ and $p 27)$ have concluded that loss of $p 16$ apparently plays a definite role in glioma progression, ${ }^{[12]}$ while low $p 27$ correlates with high-grade tumour, and predicts poor outcome. ${ }^{[13]} \mathrm{In}$ this series $p 16$ was immunonegative in $3 / 5$ cases, which was apparently comparable to that reported for conventional GBMs, ${ }^{[12]}$ p2\% immunonegativity with favourable outcome may possibly indicate that LTS cases are a separate subset of GBM, which is independent of $p 2 \%$ inhibition.

Interestingly, a gliosarcomatous transformation was observed in the giant cell GBM, which is the first such case reported in literature. This possibly represents mesenchymal metaplasia of GBM cells, ${ }^{[14]}$ rather than a separate neoplasm, which is further substantiated by the presence of $p 53$ immunopositivity in both the glial and sarcomatous areas. A vascular smooth muscle origin of the sarcoma was however ruled out by the absence of CD34 and SMA markers.

To conclude, the factors that possibly led to long-term survival in the present series could be younger age, female sex and aggressive surgical resection, in conjunction with features like focal oligodendroglial component, giant cell GBM, lack of small anaplastic cells and a low proliferation index with high p53 immunopositivity. However the role of $p 27$ and $p 16$ immunonegativity needs better definition with study of more number of eases.

This study reflects that LTS patients possibly represent a complex subset of GBMs, which cannot be characterized by a single parameter, and warrants further genetic studies. Until then, histopathologists should carefully assess GBM in young patients for foci of oligodendroglial component, giant cell ele- 
ments and areas with extensive GFAP reactivity, as well as proliferative index and p53 expression, since these appear to have prognostic connotations.

\section{References}

1. Kleihues P, Burger PC, Collins VP, Newcomb EW, Ohgaki H, Cavenee WK Glioblastoma. In: Kleihues P, Cavenee WK, editors. World Health Organization Classification of Tumours, Pathology and Geneties of Tumours of the Nervous System. Lyon, France: LARC Press; 2000;29-39.

2. McLendon RE, Halperin EC. Is the long-term survival of patients with intracranial glioblastoma multiforme overstated? Cancer 2003;98:1745-8.

3. Behin A, Hoang-Xuan K, Carpentier AF, Delattre JY. Primary brain tumours in adults. Lancet 2003;361:323-31.

4. Shinojima N, Kochi M, Hamada J, Nakamura H, Yano S, Makino K, et al. The influence of sex and the presence of giant cells on postoperative long-term survival in adult patients with supratentorial glioblastoma multiforme. J Neurosurg 2004;101:219-26

5. Schmidt MC, Antweiler S, Urban N, Mueller W, Kuklik A, Mever-Puttlitz B, et al. Impact of genotype and morphology on the prognosis of glioblastoma. J Neuropathol Exp Neurol 2002;61:321-8.

6. Klein R, Molenkamp G, Sorensen N, Roggendorf W Favorable outcome of giant cell glioblastoma in a child. Report of an 11-year survival period. Childs Nerv Syst 1998;14:288-91
7. Scott .JN, Rewcastle NB, Brasher PM, Fulton D, MacKinnon JA, Hamilton M, et al. Which glioblastoma multiforme patient will become a long-term survivor? A population-based study. Ann Neurol 1999;46:183-8.

8. Burton EC, Lamborn KR, Forsyth P, Scott J, O'Campo J, Uyehara-Lock J, et al. Aberrant p53, mdm2, and proliferation differ in glioblastomas from longterm compared with typical survivors. Clin Cancer Res 2002;8:180-7.

9. Sarkar C, Chattopadhyay P, Ralte AM, Mahapatra AK, Sinha S. Loss of heterozygosity of a locus in the chromosomal region of $17 \mathrm{p} 13.3$ is associated with increased cell proliferation in astrocytic tumors. Cancer Genet Cytogenet $2003 ; 144: 156-64$

10. Sarkar C, Ralte AM, Sharma MC, Mehta VS. Recurrent astrocytic tumors - a study of p53 immunoreactivity and malignant progression. Br .J Neurosurg $2002 ; 16: 335-42$

11. Heimberger AB, Hlatky R, Suki D, Yang D, Weinberg J, Gilbert M, et al. Prognostic effect of epidermal growth factor receptor and EGFRvIII in glioblastoma multiforme patients. Clin Cancer Res. 2005;11:1462-6.

12. Nishikawa R, Furnari FB, Lin H, Arap W, Berger MS, Cavenee WK, et al Loss of P16INK4 expression is frequent in high grade gliomas. Cancer Res $1995 ; 55: 1941-5$

13. Kirla RM, Haapasalo HK, Kalimo H, Salminen EK. Low expression of p27 indicates a poor prognosis in patients with high-grade astrocytomas. Cancer 2003; 97:644-8

14. Ozolek JA, Finkelstein SD, Couce ME. Gliosarcoma with epithelial differentiation: immunohistochemical and molecular characterization. A case report and review of the literature. Mod Pathol. 2004;17:739-45.

Accepted on 30-03-2005 